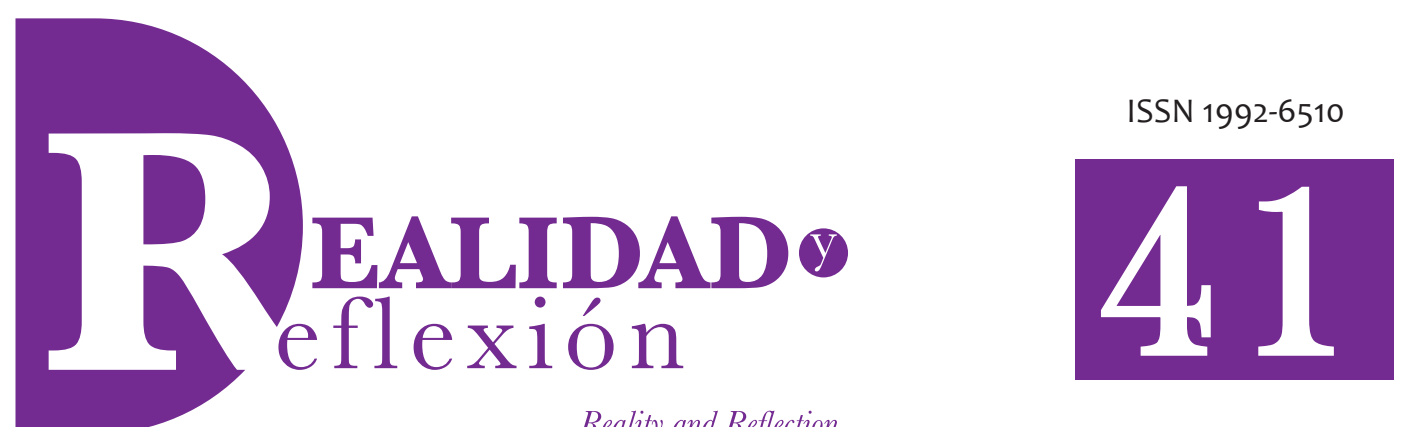

Reality and Reflection

año 15, N 41, San Salvador, El Salvador, Centroamérica Revista Semestral Enero-Junio 2015

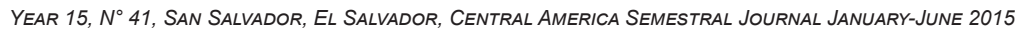

\title{
La educación superior en Santa Ana: aproximación al surgimiento de las universidades
}

\author{
Higher education in Santa Ana: \\ Approximation to the emergence of the universities
}

\author{
David E. López \\ Doctor en Filosofía y Teología \\ Instituto Bíblico de Roma \\ M.A. en Teología \\ Universidad Centroamericana José Simeón Cañas UCA \\ davidelopez@gmail.com
}

\begin{abstract}
RESUMEN
Este artículo es parte de un trabajo más amplio que realiza el Instituto de Ciencia, Tecnología e Innovación de la Universidad Francisco Gavidia. El objetivo es aproximarse a la historia de las universidades en Santa Ana. En primer lugar, se presenta una reseña de la Educación Superior en El Salvador abordándola desde sus orígenes hasta la fundación de la primera universidad en nuestro país. En segundo lugar, se documenta el surgimiento de las instituciones de educación superior en Santa Ana. La documentación apunta a que es hasta en la década de los 60 que la zona occidental cuenta con una institución educativa de nivel superior, y será hasta la década de los 80 que numerosas universidades fundan las sedes en la zona occidental. EL SALVADOR - EDUCACIÓN SUPERIOR, EDUCACIÓN UNIVERSITARIA, FORMACIÓN PROFESIONAL.
\end{abstract}

\section{ABSTRACT}

This paper is part of a wider research about history of university education in El Salvador. It contents, in first place, an overview of the beginnings of education at university level in El Salvador; in second place, the text studies the origin of upper education in Santa Ana, a western region of El Salvador. In general terms, it can be said that is not until the sixties of last century when we have notice about university education there, and it is in the first years of the eighties when some of the today universities appear in the western region of El Salvador. EL SALVADOR - HIGHER EDUCATION, UNIVERSITY EDUCATION, TRAINING. 


\section{Introducción}

La historia de la educación superior en El Salvador es una deuda que aún queda por escribirse. Bastará dar un vistazo en algunas bases de datos para concluir que son pocos y aislados los esfuerzos que sobresalen en tan necesaria labor.

En un primer término encontraremos el loable y valioso esfuerzo de Miguel Ángel Durán en construir la Historia de la Universidad de El Salvador. En dicho libro, publicado en 1941, el autor nos hace un recorrido por la universidad estatal desde 1841 hasta 1930. En segundo lugar se considera el tomo I del Diccionario histórico enciclopédico de la República de El Salvador, de Miguel Ángel García, el cual contiene una recopilación considerable de documentos concernientes a los primeros años de la universidad estatal, que comprende el período de 1841-1941. En tercer lugar podemos ubicar el ensayo de Mario Flores Macal, quien proporciona más datos desde la fundación de la primera universidad hasta inicios de la década de los 70; seguido del trabajo del Dr. Víctor Manuel Valle, quien hace un esbozo historiográfico de la Universidad de El Salvador, del surgimiento de las universidades privadas en la década de los 80 y del impacto que tuvo la guerra en estas instituciones. Por último, se consideran los Materiales para la historia de la Universidad de El Salvador, de autoría de Gregorio Bello-Suazo.

Por otra parte, lo que se puede observar es algún tipo de bibliografía respecto a las reformas constantes en la Ley de educación superior. Cabe mencionar como principales trabajos los ejecutados por Oscar Picardo Joao y su Historia y reforma de la educación superior en El Salvador, trabajo en el que aborda los antecedentes históricos de las universidades, la historia de la primera universidad en Centroamérica y el desarrollo de la educación superior en El Salvador, desde el nacimiento de la Universidad de El Salvador, el aparecimiento de las universidades privadas, hasta la explosión de estas y la crisis del sistema que culminó con la aprobación de la Ley de educación superior (Picardo, s. f.).

Enesemismo orden se encuentranartículos o informes sobre educación superior, tales como el informe presentado al Centro Interuniversitario de Desarrollo por la Dra. Maribel Duriez González y el Lic. Leonardo Coca Palacios, el cual comprende los años 2005-2010 y se plantean diversos aspectos del desarrollo institucional, tales como infraestructura institucional de provisión de la educación superior, personal docente, aseguramiento de la calidad universitaria, gobierno y gestión de las universidades, y financiamiento del sistema de educación superior.

Por último, un trabajo interesante es el de Sajid Alfredo Herrera, quien intenta mostrar que en El Salvador, durante los siglos XIX y XX, la universidad fue definida por académicos, estudiantes o autoridades universitarias a partir de los proyectos de modernización impulsados desde el interior y desde el exterior del país. Es decir, que lo que hoy se conoce como universidad fue "construido", avalando o criticando los presupuestos de los proyectos liberalespositivistas decimonónicos y los modelos desarrollistas del XX (Herrera, 2008:637).

En ese sentido, tenemos una historia fragmentada de la educación superior; 
pero que nos da algunos indicios del desarrollo de la misma. No es el objetivo de este trabajo crear una historia de la educación superior en El Salvador, sino tener un acercamiento a sus inicios en el departamento de Santa Ana.

\section{De los antecedentes de la educación superior en El Salvadr}

La formación educativa en El Salvador surge con el interés de consolidar el nuevo Estado después de la independencia. Sin embargo, en los primeros 20 años solo se logró consolidar con lentitud un modelo que se podría denominar Municipal a nivel primario (Durán, 1975:13).

En el nivel secundario, en 1825, por decreto, se manda a que se fundara el Colegio Civil y Tridentino. No obstante, de dicha fundación y funcionamiento no se ha encontrado ninguna información. En1833, se encomendó a Antonio José Coelho la fundación del Colegio "La Aurora del Salvador"; conforme el tiempo, este terminó convirtiéndose en la Escuela Normal (Durán, 1975:13-14).

Por otra parte, respecto a los intentos de fundar un centro de enseñanza superior en San Salvador, estos habían ocurrido incluso mucho antes de la independencia salvadoreña. Así pues, podemos mencionar entre ellos, el intento del Pbro. José Ignacio Ávila, quien pedía en el año de 1812 se erigiera un Seminario para la educación de la juventud, con el afán de evitar "mendigar" enseñanza en tierras distantes (Guatemala y Nicaragua).

Aproximadamente 10 años después, el diputado electo por el partido de Sonsonate, José Mariano Méndez y Cordero, pedía fundar un Centro de Cultura Superior en Cartago, Comayagua, San Salvador, Santa Ana y Quetzaltenango, con la subdirección general en Guatemala (Flores Macal 107).

Esos serían algunos intentos de fundar un centro de educación superior en nuestro país. Será hasta el 16 de febrero de 1841 que la Asamblea constituyente de El Salvador decreta se "establezca una Universidad y un Colegio de Educación". Estas instituciones se instalaron hasta el 16 de octubre de ese año en el edificio abandonado del convento de San Francisco (Durán, 1975:18-20).

El colegio se denominó La Asunción. Durante sus primeros años de trabajo se limitaron a dar clases de gramática latina y castellana. Se ha dado mucho a la confusión de que La Universidad de El Salvador y el Colegio La Asunción eran la misma institución, quizá sea porque ambos compartían el mismo edificio. Sin embargo, el Colegio desempeñaba la preparación en educación media; de hecho en 1846, 23 alumnos que se venían preparando desde 1844 reciben el grado de bachiller, para comenzar la vida universitaria propiamente dicha (Durán, 1975:23-30).

Por eso es que para el historiador Miguel Ángel Durán es acertado como inicio de la Universidad la fecha de su fundación (16 de febrero de 1841); sin embargo, es hasta el año de 1847 que inicia labores netamente universitarias con la primera promoción de 23 bachilleres (Durán, 1975:31).

\section{Hacia el surgimiento de las universidades en Santa Ana}

Por decretolegislativo, el 24 de marzo de 1965 se crea una Ley de universidades privadas. 
Esto hizo que en las décadas siguientes surgieran numerosas universidades, un total de veintinueve. ${ }^{1}$ Algunas instituciones iniciaron sus labores en la capital, creando en los próximos años filiales para la zona de occidente. Por ejemplo, la Universidad de El Salvador, en la década de los sesenta, fundó el Centro Universitario de Occidente (CUO), luego las universidades privadas también empezaron labores en la zona, tales son los casos de la Universidad Modular Abierta, la Universidad Católica de Occidente (UNICO, regentada por la diócesis de Santa Ana, y hoy por la Conferencia Episcopal de El Salvador, bajo su nuevo nombre nacional: Universidad Católica de El Salvador (UNICAES), la Universidad Nacional Autónoma de Santa Ana (UNASA) y la Universidad Francisco Gavidia (UFG).

El objetivo que subyace en esta comunicación es acercarnos al surgimiento de las universidades en Santa Ana, saber cuándo y cómo se fundaron. Para ello nos auxiliaremos de la bibliografía existente, de sus sitios web, de sus catálogos institucionales, etc.

\subsection{Universidad de El Salvador: Facultad Multidisciplinaria de Occidente}

A principios de la década de los 60 , a

\footnotetext{
1 O. Picardo considera que dado el marco legal establecido por la Ley, hubo en el país una "explosión" de veintinueve universidades en el transcurso de la década de los 80: Alberto Masferrer (1980), Evangélica de El Salvador (1981), Técnica Latinoamericana (1981), Occidental de El Salvador (1981), Tecnológica de Comercio y Administración de Empresas (1981), Francisco Gavidia (1981), Leonardo Da Vinci (1981), Nueva San Salvador (1981), Autónoma de Santa Ana (1982), Modular Abierta (1982), de Oriente (1982), Salvadoreña (1982), de Sonsonate (1982), Las Américas de El Salvador (1982), Santaneca de Ciencia y Tecnología (1982), Isaac Newton (1982), Católica de Occidente (1982), Capitán General Gerardo Barrios (1982), Pedagógica de El Salvador (1982), Americana (1982), Cristiana de las Asambleas de Dios (1983), de Educación Integral (1984), Tomás Alva Edison (1985), Don Bosco (1987), Metropolitana de El Salvador (1987), De la Paz (1987), Luterana Salvadoreña, de Administración de Negocios (1988), Panamericana (1989).
}

instancias de la Sociedad de Abogados de Santa Ana y por iniciativa de los doctores Ángel Góchez Marín, Gustavo Loyola, Luis Ernesto Arévalo y otros, se llevaron a cabo reuniones con las autoridades superiores de la Universidad; en dichas reuniones se pudo acordar que se servirían Cursos de Extensión Universitaria para el periodo de 1963 - 1964. Sin embargo, será hasta el 16 de julio de 1965 que el Honorable Consejo Universitario de la Universidad de El Salvador, según Acuerdo $N^{\circ} 46$ de la Sesión $\mathrm{N}^{\circ} 278$ (extraordinaria), conviene la creación del Centro Universitario de Occidente.

Cabe añadir que durante los primeros años de fundación del Centro, los servicios universitarios comprendían la atención del área básica o estudios generales, conocida como "Áreas comunes", de las distintas carreras y extensiones universitarias, hasta que en el año de 1971 se comenzaron a dar los servicios universitarios en las denominadas "Áreas diferenciadas"; facilitando así el complemento de carreras profesionales en el centro y, por tanto, la creación, al interior del CUO, del departamento que administrara tanto el área común como diferenciada.

La historia de la Facultad Multidisciplinaria de Occidente está cargada de intervenciones militares. La primera se realizó en 1972 y la segunda el 26 de junio de 1980. Así también, en el año de 1975 lo cuerpos militares se tomaron las instalaciones y asesinaron estudiantes que preparaban el "Desfile Bufo"; como un repudio ante tales hechos los estudiantes capitalinos salieron a manifestarse a las calles y también fueron reprimidos por los cuerpos militares, originándose así la masacre conocida y titulada como "30 de julio de 1975". 
Durante las ocupaciones las actividades académicas siguieron desarrollándose en otros locales, entre ellos cabe mencionar el Seminario Juan XXIII y una casa particular ubicada en la 9. ${ }^{a}$ Av. Sur y $11 .{ }^{a}$ calle Oriente. Es hasta el año de 1984 cuando las instalaciones son entregadas nuevamente para su funcionamiento. Sin embargo, tanto estudiantes como docentes, se involucraron en los años posteriores en distintas actividades que el contexto bélico demandaba, ocasionando así cierta irregularidad en su funcionamiento.

El año de 1992, con la llegada de los Acuerdos de Paz, también llegan nuevas disposiciones al Centro Universitario de Occidente, convirtiéndose así en Facultad Multidisciplinaria de Occidente, según acuerdo Consejo Superior Universitario $\mathrm{N}^{\circ} 39$ - 91 - 95, de fecha 4 de junio de 1992. Esto con el objeto de modernizar la estructura organizacional de la Universidad de El Salvador y perseguir con ello la mayor eficiencia en el desempeño de las actividades que el Centro Universitario llevaba a cabo.

Este nuevo cambio implicaría autonomía académica y administrativa, nuevos planes y programas, así como planificaciones y desarrollos de iniciativas de acuerdo con las necesidades de la Zona Occidental. La Facultad Multidisciplinaria de Occidente otorgó los primeros grados académicos el 26 de julio de 1992, siendo esta la primera ceremonia de entrega de títulos desarrollada en el Auditorio "Licda. Marta Pérez Cervantes", con tan solo dos meses de haberse convertido en Facultad.

En la actualidad, la Facultad Multidisciplinaria de Occidente, según su mística de trabajo, busca retomar un rumbo y una dinámica de trabajo que sitúen el quehacer académico como eje central de la actividad universitaria.

\subsection{La Universidad Católica de El Salvador}

Según el acta institucional, fue fundada el 13 de abril de 1982. El Ministerio de Educación aprobó sus estatutos, mediante el acuerdo ejecutivo $\mathrm{N}^{\circ} 1210$, de fecha 25 de junio de 1982. El Ministerio del Interior le otorgó personería jurídica, mediante el acuerdo $\mathrm{N}^{\circ}$ 279 de fecha 16 de agosto de 1982.

En 1983 se abrieron las puertas académicas a los estudiantes con la creación de la Facultad de Ciencias y Humanidades, ofreciendo 6 carreras; en 1987 se crearon las Facultades de Ciencias Agronómicas, Ciencias Económicas, Jurisprudencia y Ciencias Sociales; y en 1990, la Facultad de Ingeniería y Arquitectura. Ese mismo año se fundó el Instituto de Desarrollo Rural (IDR), en la Facultad de Ciencias Agronómicas; en 1989 se creó la Oficina de Asesoría Legal Católica (OFALCA), en la Facultad de Jurisprudencia y Ciencias Sociales; en 1990 se creó el Departamento de Educación a Distancia (DED), en la Facultad de Ciencias y Humanidades.

En 1995 se firmó un convenio con el Banco KFW de Alemania para construir el propio campus en un terreno de más de 15 manzanas, ubicado en el bypass y salida a la carretera antigua a San Salvador. En julio de 1997 la UNICAES se trasladó a su nuevo campus. Actualmente el terreno del campus tiene un área de más de 42 manzanas.

En el año 2008 el Ministerio de Educación le reconoce el cambio de denominación de Universidad Católica de Occidente por el de Universidad Católica de El Salvador, 
aprobándole los nuevos estatutos que actualmente están vigentes. Esto, dado a la apertura de un Centro Regional en Ilobasco, el cual inició labores en el año 2008. La UNICAES ha venido trabajando con el Ministerio de Educación desde el año 2006 en el modelo MEGATEC mediante el acuerdo $\mathrm{N}^{\circ}$ 15-1182, de fecha 25 de agosto de 2008, y publicado en el Diario Oficial, tomo $\mathrm{N}^{\circ} 380$, 167, de fecha 8 de septiembre de 2008.

La Universidad, por fundación, historia y práctica, está relacionada con la Diócesis de Santa Ana, que es parte de la Iglesia Católica. El hecho de que su Santidad Juan Pablo II, con fecha 18 de diciembre de 1982, a través de la Sagrada Congregación para la Educación Universitaria Católica, haya aprobado la fundación de la Universidad Católica de Occidente, hoy Universidad Católica de El Salvador, la cual es confiada jurídicamente al Obispo de la Diócesis de Santa Ana, determina la identidad católica de la Universidad.

\subsection{La Universidad Autónoma de Santa Ana (UNASA)}

La Universidad Autónoma de Santa Ana inició labores el 26 de abril de 1982. Esta Universidad fuepatrocinadaporProfesionales Santanecos S.A. de C.V., sociedad de la cual surgieron los integrantes del Consejo de Directores de la Universidad, como organismo de máxima autoridad. Sus estatutos fueron publicados en el Diario Oficial de fecha ocho de junio de 1982, e inició con un número de 161 estudiantes distribuidos en las siguientes carreras: Doctorado en Medicina, Doctorado en Cirugía Dental, Licenciatura en Psicología, Licenciatura en Trabajo Social, Licenciatura en Administración de Empresas y Licenciatura en Ciencias Jurídicas.
En 1991 se nombra al doctor Guillermo Antonio Martínez Mendoza como Rector de la Universidad, a quien se le confiere dicho cargo por su relevante participación en la Escuela de Medicina. En el año de 1993 el Consejo Superior Universitario autorizó el diseño y el financiamiento del proyecto del campus, inaugurándolo en junio de 1995 y hasta la fecha sigue funcionando, lo cual no solo incrementó el crecimiento estudiantil, sino también la calidad académica.

En consecuencia, la oferta académica se amplió en la década de los noventa, ofertando carreras como Tecnólogo en Fisioterapia, Profesorado de Educación Media para la Enseñanza de la Psicología, Profesorado en Educación Especial, Licenciatura en Ciencias de la Educación, Licenciatura en Ciencias de la Comunicación, Licenciatura en Laboratorio Clínico, Licenciatura en Enfermería y Licenciatura en Fisioterapia.

En el ámbito cultural la Universidad creó en el año 2002 el grupo de teatro "TRIFINIO", el cual por su trayectoria se convierte en el Grupo Universitario Teatral, GRUTA. En el año 2004 se fundó "RADIO UNASA", actualmente conocida como "Radio UFM 94.9". Además, se creó el periódico “El Universitario de UNASA" (2004), que posteriormente se llamó "El Informativo de UNASA", y se conoce como "EL CÉNIT".

Mientras que en el campo académico la Escuela de Postgrado, fundada en el 2004, se convierte en Dirección de Postgrado en 2009; abriendo además las áreas de Pediatría, Cirugía, y Ginecología y Obstetricia, así como la Maestría en Salud Pública. Asimismo, se fundó la Escuela de Idiomas, en el año 2006, que a partir de 2009 se denominó Unidad de Idiomas. 
La Universidad ha firmado convenios con distintas instituciones con quienes se desarrollan investigaciones, pasantías, proyectos de proyección social, actividades culturales y extracurriculares. Además, se han firmado convenios de cooperación académica con el Ministerio de Salud, hospitales, y unidades de salud, lugares donde los estudiantes ponen en práctica sus conocimientos teóricos y prácticos, que les han sido brindados en el área de la salud, por medio de sus estudios en la Universidad.

Hasta el año 2012 la Universidad contaba con 2 facultades: Ciencias de la Salud y Ciencias Sociales, 6 carreras: Doctorado en Cirugía Dental, Doctorado en Medicina, Licenciatura en Enfermería, Licenciatura en Fisioterapia, Licenciatura en Laboratorio Clínico, y Licenciatura en Ciencias de la Comunicación; contando con una población estudiantil de un mil ciento ochenta y seis $(1,186)$ estudiantes y con un total de graduados de mil ochocientos setenta $y$ nueve $(1,879)$.

\subsection{La Universidad Modular Abierta (UMA)}

Aprobados sus primeros estatutos el 26 de julio de 1982 la Universidad Modular Abierta inicias sus actividades con la idea de hacer llegar la universidad a todos los rincones del país. Aun así, inició sus labores en el año de 1981 en las ciudades de San Salvador, Sonsonate, Santa Ana y San Miguel; estableciendo en los últimos meses del mismo año otras filiales en Usulután. Posteriormente se crearon más centros universitarios en los departamentos restantes; así, se vieron involucrados los departamentos de La Paz, Chalatenango, Ahuachapán y La Unión, aunque algunos de ellos fueron vinculados a los que habían adquirido más desarrollo.

La UMA, como se conoce por sus siglas, ha formado profesionales en los grados de profesorados, tecnificados, licenciaturas y maestrías. Con la nueva Ley de educación superior, la Universidad actualizó su marco normativo legal interno, además de modificar sus planes de estudio (Cuadro 1).

\subsection{La Universidad Francisco Gavidia (UFG)}

Fundada en 1981, la Universidad Francisco Gavidia inicia sus actividades académicas el 17 de junio del mismo año. A partir de 1990 la UFG asegura su estabilidad y reconocimiento académico de todos los sectores de la sociedad, y en 1992 se crea el Centro Regional de Occidente (CRO) en la ciudad de Santa Ana.

El 12 de diciembre de 1994 se inicia la construcción del campus en San Salvador, poniéndolo al servicio el 13 de enero de 1996. Desde que la Universidad abrió sus puertas al primer grupo de estudiantes en 1981, la historia de la institución no sólo se ha escrito, sino que está reflejada en su obra física, en su trayectoria académica, en su aporte investigativo, en su contribución a la sociedad formando profesionales competentes y responsables de su desempeño, así también como en los logros obtenidos por medio de su proyección social, y su compromiso con los sectores poblacionales más necesitados.

En el año de 1999, con el afán de la búsqueda de conocimiento científico, se fundó la Unidad de Investigación y Proyección Social, que además mantiene la edición de tres revistas y el Museo Antropológico Virtual. 


\section{Cuadro 1}

\section{Modificaciones en el marco normativo de la UMA}

\begin{tabular}{lll}
\hline $\mathbf{N}^{\circ}$ de Acuerdo & Acuerdo & Fecha \\
\hline 2238 & Aprobación de primeros Estatutos Institucionales & 26 de julio de 1982 \\
485 & Aprobación de Personería Jurídica & 3 de noviembre de 1982 \\
$15-1353$ & Aprobación de Estatutos & 17 de septiembre de 1997 \\
& (Según Ley de Educación Superior) & \\
$15-0887$ & Aprobación de Nuevos Estatutos & 25 de mayo de 2005 \\
\hline
\end{tabular}

Fuente: Elaboración propia.

Tanto Theorehtikos como Societas son revistas virtuales, mientras que Realidad y Reflexión es una revista física publicada semestralmente.

La Universidad Francisco Gavidia ubica algunos periodos de mayor importancia, los cuales cubren ciclos similares en duración, pero de significado diferente. En esa línea de

\section{Bibliografía}

BELLO-SUAZO, G. (2003). Materiales para la historia de la Universidad de El Salvador. Instituto de estudios históricos, antropológicos y arqueológicos. Universidad de El Salvador.

DURÁN, M. A (1975). Historia de la universidad, 1841-1930, San Salvador: Editorial Universitaria.

DURIEZ GONZÁLEZ, M. \& COCA PALACIOS, L. (2011). La Educación Superior en El Salvador 2005- 2009. Centro Interuniversitario de Desarrollo.

FLORES MACAL, M. (1976). Historia de la Universidad de El Salvador. En Anuario tiempo, el primer período se delimita desde 1981 a 1990, el segundo incluye la transición en el lapso de 1990 a 1999, que es la época en la que funda el CRO; y el tercer periodo comienza en el año 2000 al presente.

Artículo recibido: 26 de noviembre de 2014 Artículo aprobado: 20 de diciembre 2015

de Estudios Centroamericanos de la Universidad de Costa Rica, volumen 2, pp. 107-140.

GARCÍA, M.A(1944). Diccionario histórico enciclopédico de la República de El Salvador. Universidad Nacional. Homenaje al primer centenario de su fundación. Recopilación de discursos y datos biográficos de académicos distinguidos, 1841-1941, San Salvador: Imprenta Nacional.

GONZÁLES DE MENDOZA,T. (2002). Universidad Francisco Gavidia, 20 años de historia. En Catálogo 2002 San Salvador: Universidad Francisco Gavidia. 
HERRERA, S. (2008). "El Salvador", en Carmen García Guadilla (editora), Pensadores y forjadores de la Universidad latinoamericana, Bid \& co. Editor, Venezuela, pp. 637-675.

LA UNIVERSIDAD (2013). Bosquejo histórico. 172 años de identidad universitaria. La Universidad, (20).

PICARDO JOAO, O. (s. f). Historia y Reforma de la Educación Superior en El Salvador. MINED.

UMA (2008). Catálogo Institucional. San Salvador.

UNASA (2005). Catálogo Institucional. Santa Ana.
VALLE, V.M. (1991) La Educación universitaria en El Salvador. Un espejo roto en los 80's. Centro de Investigación y Acción Social.

VARIOS (Julio de 2006). FMOcc 40 años de Servicio. Campus Occidente (2).

VARIOS (febrero 2012). 1841-2012 171 Aniversario. El universitario (19)

\section{Sitios web}

http://www.catolica.edu.sv/index.php historia/11-unicaes

http://www.ufg.edu.sv/historia.html

http://30.unasa.edu.sv/images/historia-vermas.html 